\title{
Thank You, Reviewers
}

Ann Fam Med 2010;8:186-187. doi:10.1370/afm.1096.

Peer reviewers are key to advancing scholarship and contributing to the quality of a research journal. Our sincere thanks to the following volunteers who served as peer reviewers for the Annals from October 2008 through December 2009.

Patricia Adam
David P. Adams
Alan M. Adelman
James E. Aikens
Deborah I. Allen
Suzanne M. Allen
Bhavana Anand
Gowri Anandarajah
J. Ellen Anderson
David C. Anthony
Nedal H. Arar
Puneet S. Arora
Bruce Arroll
Brian J. Ayotte
Bruce A. Bagley
Seong-Yi Baik
Megan H. Bair-Merritt
Bijal A. Balasubramanian
Beth Barnet
Michael S. Barr
Bruce P. Barrett
James E. Barrett
Henry C. Barry
Claudia C. Bartz
Dennis J. Baumgardner
Andrew W. Bazemore
John W. Beasley
Lorne A. Becker
Justin Beilby
Kristen L. Bene
Ian M. Bennett
Diana M. Bensyl
Margo Bergman
Leonard L. Berry
Cheryl A. Bettigole
Krishna V. Bhaskarabhatla
Kavitha Bhat-Schelbert
Linda T. Bilheimer
Lyla J. Blake-Gumbs
Robert P. Blankfield
Alan M. Blum
Thomas Bodenheimer
Francesc Borrell-Carrio
Hayden B. Bosworth
David M. Bott
Carol A. Bova
Peter Bower
Rebecca S. Boxer
Raymond G. Boyle
Elizabeth J. Bragg
Robert L. Bratton
Helena C. Britt
Jane E. Brock
Stephen A. Buetow

Sandra K. Burge
Jochen W. Cals
James F. Calvert Jr
Lucy M. Candib
Roberto Cardarelli
Matthew J. Carlson
Teresa L. Carman
Michael A. Carter
Lawrence P. Casalino
Anthony P. Catinella
Bruce A. Center
Robert M. Centor
Rajesh Chauhan
Frederick M. Chen
Ping-Hsin Chen
Cynthia Cheng
Kai Ming Chow
Mary-Margaret Chren
Charles E. Christianson
Dana L. Clark
Elizabeth C. Clark
Lynn P. Clemow
Andrew S. Coco
Simon Cohn
Katie Coleman
Douglas A. Conrad
W. Carl Cooley
Anthony J. Costa
Glen R. Couchman
Ben Crabtree
Jennifer Creer
Jesse C. Crosson
Brian K. Crownover
Larry Culpepper
Dosha F. Cummins
Sue Curry
William J. Curry
Timothy P. Daaleman
Barbara J. Daly
Jeanette M. Daly
Elvan C. Daniels
Sean P. David
Richard G. Davies
Esa Davis
An De Sutter
Frank V. deGruy
Renee M. DeHart
George L. Delclos
Dianne Delva
Kelly J. Devers
Myriam Deveugele
Jennifer E. DeVoe
Barbara DiCicco-Bloom
Lori M. Dickerson

L. Miriam Dickinson
W. Perry Dickinson
Allen J. Dietrich
Mary Dixon-Woods
Susan M. Dovey
Patrick T. Dowling
Richard L. Dressler
Michael F. Dulin
Anne L. Dunlop
Charles B. Eaton
Justin B. Echouffo Tcheugui
Thomas R. Egnew
Nancy C. Elder
Stephen D. Elgert
John W. Ely
J. C. Herbert Emery
Bery J. Engebretsen
John W. Epling
Ronald M. Epstein
Rebecca S. Etz
Bernard Ewigman
Linda J. Ewing
Lyle J. Fagnan
Steven G. Federico
Chris Feifer
Michael F. Felder
Douglas H. Fernald
Jeanne M. Ferrante
Michael D. Fetters
Deborah Finfgeld-Connett
Kenneth S. Fink
Susan A. Flocke
Grace E. Flood
Colleen T. Fogarty
Joshua Fogel
Samuel N. Forjuoh
Luke W. Fortney
Beth A. Fox
Chester H. Fox
Peter Franks
Crystal M. Freeman
Joshua Freeman
Jan C. Frich
Dominick L. Frosch
George E. Fryer
Kelly Fryer-Edwards
Bridget Gaglio
James Galliher
Joseph J. Gallo
Theodore G. Ganiats
Patricia A. Gately
Leon N. Geffen
Lillian Gelberg
Jochen S. Gensichen

Darios Getahun
Michael D. Geurin
Mark D. Gideonsen
Valerie J. Gilchrist
James M. Gill
William R. Gillanders
Charles (Bill) W. Given
Russell E. Glasgow
James L. Glazer
Emily M. Godfrey
Marshall Godwin
Amanda L. Golbeck
Marji Gold
Marthe R. Gold
Debora G. Goldberg
Roberta E. Goldman
Ana I. Gonzalez
Felicity A. Goodyear-Smith
Indiran Govender
Jonathan P. Graffy
Robert E. Gramling
Lars-Petter Granan
Larry A. Green
Lee A. Green
Michael L. Grover
Kevin Grumbach
Janelle M. Guirguis-Blake
Jane M. Gunn
Natalia Gutierrez-Chefchis
Jeannie Haggerty
David L. Hahn
Ricardo G. Hahn
Susan Hart-Hester
Marc I. Harwood
Stephen E. Hawes
Iona Heath
Diana L. Heiman
Lynn D. Helseth
Margaret R. Helton
Eric Henley
John M. Hickner
Paul C. Hicks
David C. Hodgson
John H. Holmes
James D. Holt
Jodi Summers Holtrop
Jennifer L. Hoock
Catherine Hudon
Melissa M. Hudson
William J. Hueston
Mark K. Huntington
Belinda K. Ireland
Noah M. Ivers
Douglas J. Ivins 
Eric A. Jackson

Carlos R. Jaen

Paul A. James

Verlin K. Janzen

Mollie W. Jenckes

Anthony F. Jerant

Jane C. Johnson

Glenn N. Jones

Nora L. Jones

Linda S. Kahn

Tina L. Karwalajtys

David A. Katerndahl

Robert D. Keeley

Amanda J. Keerbs

Anthony R. Kendrick

Ngaire Kerse

Niharika Khanna

Morteza Khodaee

Sanford R. Kimmel

Dana E. King

Valerie J. King

Dorte Kjeldmand

Lyndee Knox

Mark A. Knox

Michael M. Kochen

Francis P. Kohrs

Richelle J. Koopman

Neil Korsen

Richard L. Kravitz

Alex H. Krist

Robin L. Kruse

David Kuo

Grace M. Kuo

Anton J. Kuzel

Jennifer Elston Lafata

Judy Lalmuanpuii

Steven H. Landers

JoAnn E. Leavey

Michael L. LeFevre

Sarah E. Lesko

Jennifer B. Levin

Barcey T. Levy

$\mathrm{Li} \mathrm{Li}$

Jane Liebschutz

Elizabeth H. Lin

Kenneth W. Lin

Cristos Lionis

David G. Litaker

Everett E. Logue

Daniel R. Longo

Daniel S. Longyhore

Margaret M. Love

David A. Loxterkamp

Paula Lozano

Sean C. Lucan

Peter Lucassen

Ann C. Macaulay

Michael V. Maciosek

Diane J. Madlon-Kay

Julie M. Magri

Manfred Maier

Deborah S. Main

Arch G. Mainous III

Barbara A. Majeroni

Rebecca A. Malouin
Kirsti Malterud

Rita Mangione-Smith

Lucille R. Marchand

Ruta S. Marfatia

Mary M. Marfisee

William H. Markle

Mark R. Marnocha

Larry B. Mauksch

Nahal Mavaddat

Patrick E. McBride

John R. McConaghy

Julie J. McGowan

Warren J. Mclsaac

James F. McKenzie

Robert K. McKinley

David E. McRay

Cliff A. Megerian

Ateev Mehrotra

Tai J. Mendenhall

Michael D. Mendoza

Mark B. Mengel

Stewart W. Mercer

Dan J. Merenstein

David S. Meyers

J. Lloyd Michener

Jennifer L. Middleton

Benjamin F. Miller

William L. Miller

Todd D. Miller

W. Fred Miser

Arvind Modawal

James W. Mold

L. Gordon Moore

Nancy E. Morden

Gerardo Moreno

Masahiro Morikawa

Christopher P. Morley

Cynthia D. Morris

David J. Muzina

Marion R. Nadel

Zsolt J. Nagykaldi

Sudip Nanda

Ira S. Nash

Konrad C. Nau

Irwin Nazareth

Donald E. Nease Jr.

Jon O. Neher

Warren P. Newton

J. Michael Niehoff

Paul J. Nietert

Thomas E. Norris

Mary Patricia Nowalk

Patrick J. O'Connor

Kevin C. Oeffinger

Pamela A. Ohman Strickland

Ardis L. Olson

Steven M. Ornstein

Ramnarayan Paragi-Gururaj

Michael L. Parchman

Donald E. Pathman

Ronald A. Paulus

Lars E. Peterson

Julie P. Phillips

Robert L. Phillips

Kathryn E. Phillips
David Pierce

Marty S. Player

Donna J. Plonczynski

Anita Pomerantz

Edward P. Post

Michael B. Potter

Shailendra Prasad

Richard W. Pretorius

David W. Price

Sandra Puczynski

Wayne Putnam

Nadeem Qureshi

Howard K. Rabinowitz

Lee J. Radosh

Kalyanakrishnan Ramakrishnan

Brian V. Reamy

Sean W. Reed

Ann B. Reichsman

Jacob M. Reider

Shmuel P. Reis

William H. Replogle

Caroline R. Richardson

Alfred A. Rimm

J. Adam Rindfleisch

Cynthia M. Ripsin

Manfredi Rizzo

Mary B. Roberts

W. David Robinson

Donna L. Rochon

William M. Rodney

Richard G. Roetzheim

John C. Rogers

James E. Rohrer

Martin Roland

Gerald M. Rosen

Ellen Rosenberg

Thomas C. Rosenthal

Walter W. Rosser

Danica Rotar Pavlic

Stephen F. Rothemich

Grant M. Russell

Chris Salisbury

Margarete Sandelowski

Simon P. Sanderson

Robert B. Saper

John W. Saultz

Judith K. Schaefer

Bernhard J. Schaller

Joseph E. Scherger

Julie A. Schmittdiel

Ronald Schneeweiss

Michael D. Schooff

Sarina B. Schrager

Herbert C. Schulberg

Stephen E. Schuldt

Thomas L. Schwenk

John G. Scott

Dean A. Seehusen

Allen F. Shaughnessy

Eric K. Shaw

Brian M. Shelley

Todd M. Sheperd

Judy C. Shlay

Kerem Shuval

Bonnie Sibbald
Randa D. Sifri

Laura A. Siminoff

Christine A. Sinsky

Keith S. Sinusas

Philip D. Sloane

Mindy A. Smith

Robert W. Smith

William D. Smucker

Maria Rosa Solorio

Jeffrey H. Sonis

Barbara Starfield

Howard F. Stein

Elizabeth Steiner

John F. Steiner

Mary M. Step

Nancy G. Stevens

Elizabeth E. Stewart

Christopher J. Stille

Richard Streiffer

Jeff L. Susman

Andrew L. Sussman

Alfred F. Tallia

Stephen H. Taplin

Carolyn Tarrant

Jonathan L. Temte

David H. Thom

Paul Thomas

Jeffrey D. Tiemstra

Elizabeth M. Tracy

David H. Trump

Fred G. Tudiver

James W. Tysinger

Mark E. Unverzagt

Brian K. Unwin

Jose M. Valderas Jr.

Anje C. Van Berckelaer

Mieke L. van Driel

Daniel J. Van Durme

Eric van Rijswijk

Chris van Weel

Theo Verheij

Mark E. Vogel

Howard Waitzkin

Lorraine S. Wallace

Jeanette A. Waxmonsky

Brian J. Wells

William (Bill) G. Weppner

James J. Werner

David R. West

John M. Westfall

Lisa C. Whitehead

Simon N. Whitney

Ellen Wiebe

Robert L. Williams

John J. Wilson

Samuel Y. Wong

J. Susie Woo

Douglas C. Woolley

Jinping $\mathrm{Xu}$

Barbara P. Yawn

Amanat (Amy) M. Yosha

Therese M. Zink

Adam Zolotor

Kathy Zoppi

John P. Zubialde 